\title{
Unusual presentations of chemotherapy induced acral erythema after high dose methotrexate: Case series
}

\author{
Anant Vatsayan ${ }^{1 *}$, Hasan Hashem ${ }^{1}$, Kord Honda ${ }^{2}$, Rachel Egler ${ }^{1}$ and Robin Norris ${ }^{1}$ \\ ${ }^{1}$ UH Rainbow Babies and Children's Hospital, Department of Pediatric Hematology/Oncology 11100, Euclid Avenue, Cleveland, Ohio-44106, USA \\ ${ }^{2}$ University Hospitals, Case Medical Center, Department of Dermatology, 11100, Euclid Avenue, Cleveland, Ohio-44106, USA
}

\begin{abstract}
Typically, chemotherapy induced acral erythema (CIAE) presents with bilaterally symmetrical dysesthesia followed by the development of erythematous plaques on palms and soles after exposure to chemotherapy. We report 2 pediatric patients with atypical presentations (predominantly unilateral) of CIAE after treatment with high dose methotrexate (HD MTX). These cases highlight the unusual manifestations of CIAE that may confound diagnosis and delay appropriate treatment.
\end{abstract}

\section{Case reports}

\section{Patient 1}

A 15 year-old female was being treated for mixed lineage leukemia (predominant T cell phenotype) according to the Children's Oncology Group (COG) protocol AALL0434. During induction, she developed left hemiparesis secondary to sagittal sinus venous thrombosis and right intraparenchymal hemorrhage. On Day 1 of interim maintenance (IM), she was started on $5 \mathrm{gm} / \mathrm{m}^{2}$ of HD MTX. Serial MTX levels showed appropriate clearance with normal creatinine levels. Forty-two hours after starting HD MTX infusion, leucovorin (LCV) was started and continued until MTX level was $<0.4 \mu \mathrm{mol} / \mathrm{L}$. At 72 hours, she complained of pain with tingling in her left elbow and dorsum of her left foot followed by development of erythematous, well demarcated tender plaques (Figure 1A, 1B). A desquamating erythematous patch was also noted in the left axillary region abutting the area of her brassiere band (Figure 1C). Skin biopsy obtained from the left elbow lesion was consistent with CIAE (Figure 2). Topical application of cold packs, diclofenac gel, lidocaine patch $(5 \%)$ and oral Celecoxib had minimal response. Her symptoms gradually resolved over the next 2 weeks. For all subsequent doses of HD MTX, she received $5 \mathrm{gm} / \mathrm{m}^{2} /$ dose with $200 \mathrm{~mL} / \mathrm{m}^{2} / \mathrm{h}$ hydration and $\mathrm{LCV}$ rescue $\left(15 \mathrm{mg} / \mathrm{m}^{2} /\right.$ dose $)$ until her MTX level was $<0.1 \mu \mathrm{mol} / \mathrm{L}$, with no recurrence of CIAE.

\section{Patient 2}

A 16 year-old female with a history of histiocytic sarcoma and
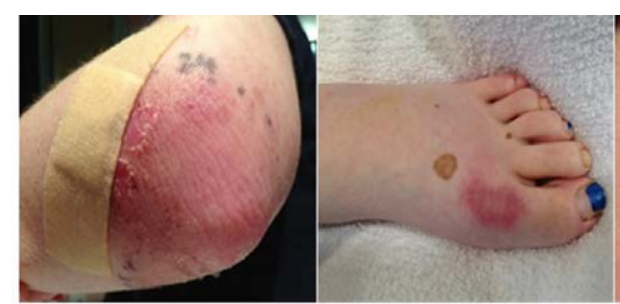

A

B

\section{C}

Figure 1. Patient 1: Well demarcated erythematous plaques on the left elbow (A), foot (B) and chest $(\mathrm{C})$.
Raynaud's disease was being treated for very high-risk precursor B cell acute lymphoblastic leukemia (ALL) according to COG protocol AALL1131. Two days prior to starting the third cycle of HD MTX in IM 1, she had an episode of Raynaud's disease, predominantly involving the right hand. On day 1 of this cycle she was started on $5 \mathrm{~g} /$ $\mathrm{m}^{2} /$ dose of HD MTX infused over 24 hours with hydration fluid rate of $200 \mathrm{~mL} / \mathrm{m}^{2} /$ hour due to previous delayed MTX excretion. Serial MTX levels showed appropriate clearance. LCV was started 42 hours after initiation of HD MTX and was continued until MTX level was $<0.4$ $\mu \mathrm{mol} / \mathrm{L}$. One day after starting HD MTX, she experienced worsening of erythematous rash over the bilateral palms which was more prominent on the right side (Figure 3). Rash was now accompanied by pain and tingling. Based on the history and dermatologic manifestations a clinical diagnosis of CIAE was made. No confirmatory biopsy was performed since it was deemed unnecessary. Significant improvement was noticed after 2 days of topical application of eucerin and triamcinolone cream

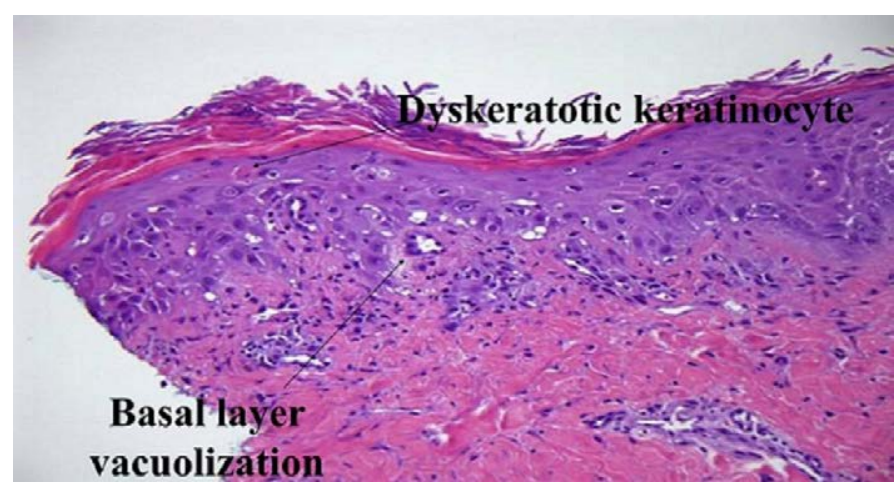

Figure 2. Patient 1: Hematoxylin and eosin stained section of skin lesion showing dyskeratotic keratinocytes, vacuolar changes and dysmaturation of epidermal keratinocytes.

Correspondence to: Anant Vatsayan, UH Rainbow Babies and Children's Hospital, Department of Pediatric Hematology/Oncology 11100, Euclid Avenue, Cleveland, Ohio-44106, USA Tel: 216-844-3345; E-mail: vatsayan25@gmail.com

Key words: botulinum toxin, generalized dystonia, deep brain stimulation

Received: May 10, 2017; Accepted: May 23, 2017; Published: May 26, 2017 


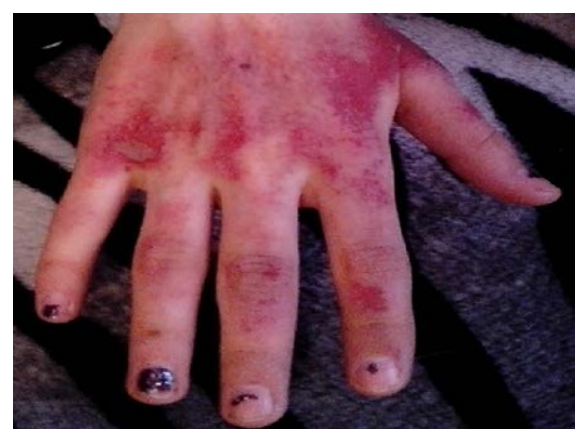

Figure 3. Patient 2: Well demarcated erythematous areas over the dorsum of the right hand.

(0.1\%). 5 days after starting HD MTX, mild desquamation was noted with complete resolution of the rash in next 1 week. No methotrexate dose modifications were made in subsequent courses and the patient did not have any recurrence.

\section{Discussion}

CIAE has a heterogenous clinical presentation. However, it typically develops between 1 day to 3 weeks after drug exposure, with a prodrome of dysesthesia followed by bilaterally symmetric, erythematous plaques involving palms and/or soles. The rash is usually associated with mild swelling and desquamation. Symptoms usually resolve within 1 to 3 weeks of its appearance [1]. Histopathology may show dyskeratosis, vacuolation and spongiosis in the epidermis with perivascular lymphohistiocytic infiltrate, syringosquamous metaplasia, or eccrine neutrophilic hidradenitis [2]. While numerous chemotherapeutic drugs can cause CIAE, most cases in children have been described in association with HD MTX [3]. Purportedly, areas with a high density of eccrine sweat glands have higher concentration of MTX, supporting the predisposition of the palms and soles. Trauma, frequent friction and pressure over body parts may lead to extravasation of inciting drugs into surrounding tissue, resulting in local toxicity. MTX is known to accumulate in edematous tissues. Decreased systemic clearance of MTX can also increase the likelihood of CIAE [1,2]. It is possible that Patient 1 had dependent edema on the paretic side, predisposing that side to the accumulation and toxic effects of MTX. Interestingly, topography of the rash corresponded to the areas predisposed to repeated microtrauma and friction. The erythematous patch on the left chest wall aligned with the band of brassiere while the rash over left elbow could be due to repeated friction from bearing weight on the paretic side while lying in bed or getting up. Additionally, the areas on her left hand and foot coincided with the application of wrist and foot braces. Similarly, the prominence of CIAE in the right hand in Patient 2 may be explained by extravasation of MTX, predominantly on the right side due to asymmetric involvement of her hands by Raynaud's disease.

Most cases of CIAE resolve spontaneously and there is lack of clear evidence delineating the proper management of CIAE. Early detection and management is critical to avoid progression to debilitating symptoms, including bullous eruptions. Preventive strategies include application of emollients/moisturizer besides avoiding exposure to extreme temperature and trauma/friction to the predisposed areas. Treatment interruption and interval prolongation between consecutive chemotherapy may be effective while subsequent dose reductions have questionable benefit $[2,4]$. Frequently used interventions include cold packs, steroid creams, and emollients. Oral treatment with NSAIDS, pyridoxine and steroids have shown inconsistent results $[1,2,4,5]$.

The differential diagnosis may include trauma, allergic reactions, cellulitis, Sweet's syndrome and juvenile Still's disease. Bullous presentations may mimic staphylococcal scalded skin syndrome, Steven-Johnson Syndrome, paraneoplastic pemphigus or radiation dermatitis $[1,5,6]$. In conclusion, awareness about the protean manifestations of CIAE in patients undergoing chemotherapy is key to diagnosis. Usually, biopsy is unnecessary and the diagnosis is based on recognizing dermatologic manifestations of CIAE following exposure to chemotherapeutic drugs.

\section{Acknowledgement}

This work required no support from any funding agency or pharmaceutical industry.

\section{References}

1. Miller KK, Gorcey L, McLellan BN (2014) Chemotherapy-induced hand-foot syndrome and nail changes: a review of clinical presentation, etiology, pathogenesis, and management. J Am Acad Dermatol 71: 787-794.[Crossref]

2. Degen A, Alter M, Schenck F, Satzger I, Völker B, et al. (2010) The hand-footsyndrome associated with medical tumor therapy - classification and management. $J$ Dtsch Dermatol Ges 8: 652-661.[Crossref]

3. Millot F, Auriol F, Brecheteau P (1999) Acral erythema in children receiving high-dose methotrexate. Pediatr Dermatol 5: 398-400.

4. Farr KP, Safwat A (2011) Palmar-plantar erythrodysesthesia associated with chemotherapy and its treatment. Case Rep Oncol 4: 229-235.

5. Webster-Gandy JD, How C, Harrold K (2007) Palmar-plantar erythrodysesthesia (PPE): a literature review with commentary on experience in a cancer centre. Eur J Oncol Nurs 11: 238-246.

6. Aytaç S (2010) Acral erythema with bullous formation: a side effect of chemotherapy in a child with acute lymphoblastic leukemia. Turk J Pediatr 52: 211-214.

Copyright: (C2017 Vatsayan A. This is an open-access article distributed under the terms of the Creative Commons Attribution License, which permits unrestricted use, distribution, and reproduction in any medium, provided the original author and source are credited. 